\title{
10. SUB-SYSTEM OF NOVAE
}

\author{
I. M. KOPYLOV \\ Crimean Astrophysical Observatory, Pochtovoje, Crimea, U.S.S.R.
}

In order to discover the cause of the outbursts of novae, a study of the physical processes taking place in novae during such explosions is not sufficient. A number of other, and no less important, properties and peculiarities of novae must also be investigated. Such properties are, for instance: the absolute magnitudes at light maximum and minimum, the temperatures, the light curves and their variations, the position of the novae on the Hertzsprung-Russell diagram, their spatial distribution in the Galaxy, and the mutual relations between the novae and other objects of similar nature (such as the recurrent novae, super-novae, nova-like stars, and the nuclei of planetary nebulae). A hypothesis of the origin of the outbursts of novae should be founded upon all these data as well as the other properties of novae.

A knowledge of the absolute magnitudes of novae at light maximum $\left(M_{\text {max. }}\right)$ is required for the solution of a number of questions on the physical properties of novae.

The most reliable method for a determination of the absolute magnitudes of novae (from the expansion of the ejected envelopes) permitted McLaughlin in I945 to define (with the aid of some other methods and with allowance for space absorption) the relationship between the absolute magnitude of a nova at light maximum, $M_{\text {max. }}$, and the time $t_{3}$ in days of the decline of the light of the nova for 3 magnitudes after the light maximum. The dependence $\left(M_{\text {max., }}, t_{3}\right)$ (see Fig. I) can be expressed by the equation:

$$
\begin{aligned}
M_{\text {max. }}= & -\mathrm{I} 3^{\mathrm{m} \cdot 7}+3^{\mathrm{m} \cdot 6 \log t_{3}} \\
& \pm 0 \cdot \mathrm{I} \quad \pm 0 \cdot 2
\end{aligned}
$$

The absolute magnitude of a nova at light maximum can thus be determined according to the value of $t_{3}$ from the light curve. Equation (I) affords a most simple method for the determination of the absolute magnitudes, and, consequently, of the distances of the novae.

A study of the light curves of novae permits one to establish the following two relations:

(a) Immediately after maximum the light of a nova declines according to the law:

$$
M_{i}=-\mathrm{I} 3^{\mathrm{m} \cdot 7}+3^{\mathrm{m} \cdot 5} \log t_{3}^{i}
$$


where $M_{i}$ is the absolute magnitude of the nova at a given moment (at the moment of light maximum in particular), and $t_{3}^{i}$ is the time of the subsequent decline of its light for 3 magnitudes.

(b) The duration of the total decline of the light of a nova from maximum to minimum is determined by the luminosity of the nova at maximum.

These two peculiarities of the light curves of novae express, in fact, the same important general property of the novae: the rate of the subsequent

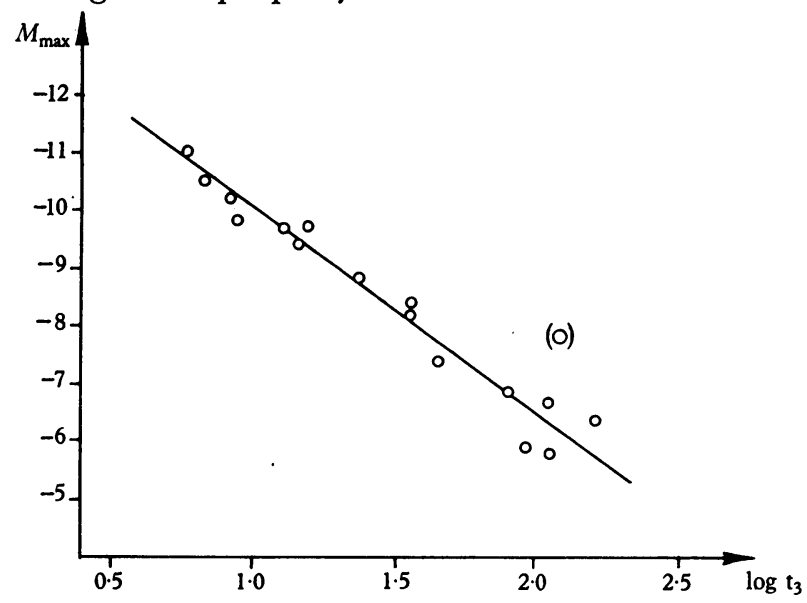

Fig. I. The dependence of the absolute magnitude of novae at maximum light upon the time (in days) required for the light to decline 3 magnitudes below maximum.

decline of the light of a star is determined by the value of its luminosity at a given moment. Similar relationships between phenomena and the rates of their evolution can also be observed in some other astrophysical processes. For example, they appear in the outbursts of super-novae, the development of the chromospheric flares on the Sun, and so on.

The relation (2) permits one to determine the absolute magnitude of a nova from any part of the light curve where the luminosity of the star decreases not less than 3 magnitudes. This permits one to extend considerably the data regarding the absolute magnitudes and distances of novae, using only their light curves.

The luminosity function of novae at light maximum obtained in the first approximation has the following parameters: a mean value of $M_{\max .}=-7^{\mathrm{m}} \cdot 5$, extreme values of $-2^{\mathrm{m} \cdot 0}$ and $-\mathrm{I}^{\mathrm{m}} \cdot \mathrm{o}$, and a dispersion of $\pm 2^{\mathrm{m} \cdot} \cdot 5$.

The luminosity function of novae in their normal state has these parameters: a mean value of $+3^{\mathrm{m} \cdot \mathrm{I}}$, extreme values of $-\mathrm{I}^{\mathrm{m} \cdot} \cdot 5$ and $+7^{\mathrm{m} \cdot} \cdot 5^{\text {, and }}$ a dispersion of $\pm 2^{\mathrm{m}} \cdot 5$. The parameters of the distribution of novae accord- 
ing to the values of their amplitudes are: a mean value of ${ }_{10}{ }^{\mathrm{m}} \cdot 4$, extreme values of $6^{\mathrm{m} \cdot \mathrm{o}}$ and $\mathrm{I} 5^{\mathrm{m} \cdot \mathrm{o}}$, and a dispersion of $\pm 3^{\mathrm{m} \cdot 5}$.

$A$ comparison with super-novae. The faintest super-novae (about $M=-13$ at light maximum) are approximately 2 magnitudes brighter than the most luminous novae (of about $M=-$ I I ). The absence of exploding stars of intermediate absolute magnitudes (from -1 I to $-13^{m}$ ) is obviously real. The mean absolute magnitude of super-novae at light maximum is about $-15^{\mathrm{m} \cdot 7}$, and of typical novae only about $-7^{\mathrm{m} \cdot 5}$.

The amplitudes of the super-novae are not less than 21 magnitudes. The largest amplitudes of the novae can, apparently, reach but 15 to 17 magnitudes.

For super-novae as well as for novae, a relation $\left(M_{\max .}, t_{3}\right)$ holds true. This relation for super-novae is expressed by the equation

$$
\begin{aligned}
M_{\text {max. }}= & -3^{\mathrm{m} \cdot} \cdot 9+9^{\mathrm{m} \cdot} \cdot 5 \log t_{3} \\
& \pm 0 \cdot 3 \quad \pm 0 \cdot 2
\end{aligned}
$$

It is easy to see that the relation $\left(M_{\text {max. }}, t_{3}\right)$ for super-novae is not an extension of the similar relation (I) for typical novae. The spatial distribution of novae and super-novae in the Galaxy is also altogether different. The super-novae form a flat sub-system, while the typical novae form an intermediate sub-system. Thus the novae and super-novae form two independent groups of stars, clearly differing both in the distribution of their amplitudes and absolute magnitudes at maximum, as well as in their distribution in the Galaxy.

A comparison with the recurrent novae and U Geminorum type stars. The novae were previously considered to form an extension of the recurrent novae towards the region of large amplitudes and large periods. This assumption was based upon the 'amplitude-cycle' relation, which was established for the nova-like U Geminorum type stars. Recurrent novae were assumed to satisfy this relation, as well as were the typical novae. But the accumulation of observational data concerning stars of these types shows quite clearly intrinsic differences in the physical properties, as well as in the mechanisms of the outbursts, between the nova-like stars and the recurrent novae.

It was found also that the 'amplitude-cycle' relation is different for both groups of stars. The 'amplitude-cycle' relation for nova-like stars is

$$
\log P=-0.38+0 \cdot 60 A,
$$

and for the recurrent novae is

$$
\log P=+2 \cdot 83+0 \cdot 14 A,
$$

where $A$ is the amplitude and $P$ is the period expressed in days (Fig. 2). 
A comparison of the amplitudes of the typical and recurrent novae shows that the novae do not satisfy relation (5), established for the recurrent novae, because the typical novae do not explode a second time, in spite of having approximately the same amplitudes as do the recurrent novae. Since repeated outbursts of novae have never been observed, the question of the intervals between such bursts remains open. It is quite possible that outbursts of typical novae occur only once in the course of their lifetime. It should be remembered in this connexion that all the statistics concerning

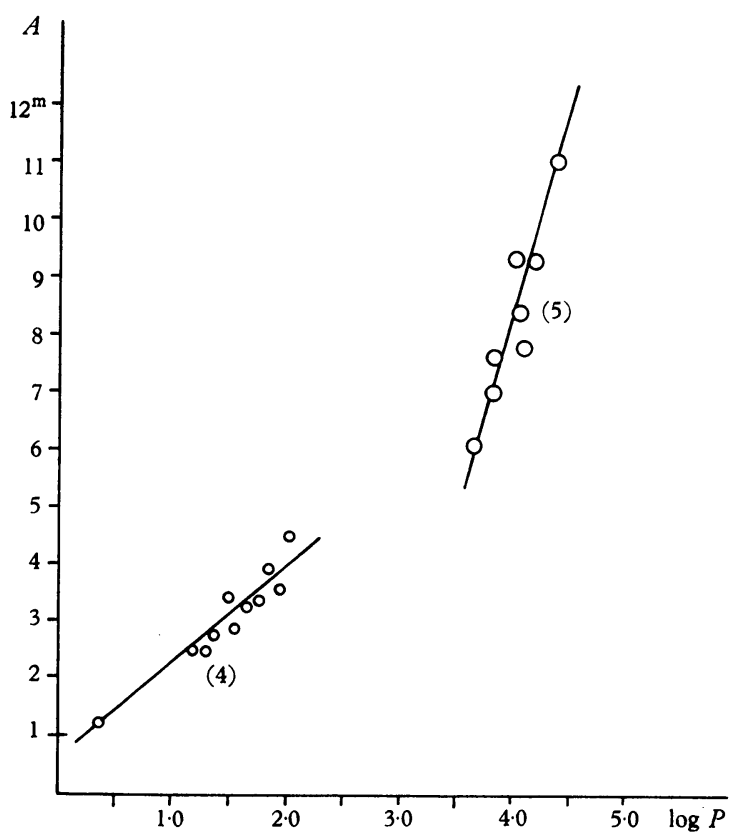

Fig. 2. The dependence of amplitude upon period for the nova-like U Geminorum variables (the straight line is that of equation (4)) and for the recurrent novae (the line is that of equation (5)).

the numbers of novae in the Galaxy are based upon their frequency in the present stage of the Galaxy.

The recurrent and typical novae form sub-systems of different flatness in the Galaxy.

The data quoted, together with additional information, permit one to suggest that there is no genetic connexion between the typical and recurrent novae and that they represent two altogether different types of stars.

A comparison of novae with the nuclei of planetary nebulae. A common feature of the novae and the nuclei of the planetary nebulae is the presence of expanding envelopes. The envelopes of novae have smaller masses $\left(\mathrm{IO}^{-3}\right.$ to $\left.\mathrm{Io}^{-4} \mathbf{m} \odot\right)$ and the velocity of their expansion is larger $(v \sim$ Iooo $\mathrm{km} . / \mathrm{sec}$. 
than in the case of planetary nebulae $\left(\mathrm{m} \sim \mathrm{IO}^{-1}\right.$ to $1 \mathrm{O}^{-2} \mathbf{m} \odot$, and $v \sim \mathrm{Io} \mathrm{km} . /$ sec.).

Novae (in their normal state) and the nuclei of the planetary nebulae occupy about the same region in the spectrum-luminosity diagram, namely that of high temperatures and moderate luminosities. The luminosity functions of the novae and of the nuclei of planetary nebulae show a close similarity: $\bar{M}_{\mathrm{min} \text {. }}=+3 \cdot \mathrm{I}$ for novae and $\bar{M}=+2 \cdot 5$ for the nuclei. The extreme values of the absolute magnitudes are $-2^{\mathrm{m}}$ and $+8^{\mathrm{m}}$ for novae, and $-2^{\mathrm{m}}$ and $+7^{\mathrm{m}}$ for the nuclei of planetary nebulae. Thus, they are practically coincident. The spectra and, consequently, the temperature of the novae (in their normal state) and of the nuclei of planetary nebulae are also practically the same.

The apparent distribution in the sky of novae and planetary nuclei is rather similar. Stars of these two types form intermediate sub-systems with practically identical parameters. Their mean spatial velocities are also similar. From such a comparison of the novae with stars of other types, it follows that the nuclei of planetary nebulae show the closest similarity to the novae.

On the basis of these results, the following may be said about the origin and evolution of novae. In accordance with the ideas of VorontsovVelyaminov, who supposes an evolution of stars along the blue-white sequence, it appears that the novae represent an intermediate stage between the recurrent novae and the white dwarfs.

The differences that have been pointed out between these stars make the transfer of the recurrent novae into the typical novae highly improbable, if the following two circumstances in particular are considered.

(a) The novae do not satisfy the amplitude-cycle relation for the recurrent novae, and $(b)$, they form a sub-system in the Galaxy totally different from that of the recurrent novae.

These two types of stars are, probably, of quite different origin and age.

According to rather rough estimates $\bar{M}_{\text {min. }}=+2 \cdot 9$ for the recurrent novae, $\bar{M}_{\mathrm{min} .}=+3 . \mathrm{I}$ for the typical novae and $\bar{M}=+2.5$ for the nuclei of planetary nebulae. All these three types of stars are extremely hot objects. Thus, stars of these types, which eject (in some way or other) discrete envelopes, are located in the same region of the luminosityspectrum diagram. It may be admitted that the evolution of such stars follow, in general, parallel courses, but nothing conclusive can at present be said regarding the final stage of evolution of novae. We are not inclined to exclude completely the possibility that the white (or blue) dwarfs are the final stage of the development of novae, since the parameters of their 
sub-systems are very similar. However, the absence of blue and white stars in the range of absolute magnitude between +7.5 and $+9^{\mathrm{m}} \cdot 5$ decreases this possibility immensely, such a gap being quite incomprehensible in terms of the hypothesis of the evolution of novae into white dwarfs.

From all the foregoing results it follows, in any case, that the interpretation of the blue-white sequence as an evolutionary track of hot giant stars should be altogether rejected.

A table containing the parameters of the spatial distribution in the Galaxy of novae and the objects that are to some extent related with novae is given below:

Galactic Distribution Parameters for Super-novae, Novae, Planetary Nuclei, and Similar Objects

\begin{tabular}{|c|c|c|c|}
\hline Sub-system & $\frac{\partial \log D}{\partial R}$ & $\frac{\partial \log D}{\partial z}$ & $\begin{array}{c}\beta \\
\text { (parsecs) }\end{array}$ \\
\hline Super-novae & $-0 \cdot 15$ & $-5 \cdot 2$ & 83 \\
\hline Nuclei of planetary nebulae & -0.21 & $-2 \cdot 20$ & 197 \\
\hline Typical novae & -0.22 & $-2 \cdot 39$ & 182 \\
\hline White dwarfs & $-0 \cdot 23$ & $-2 \cdot 7$ & 160 \\
\hline Recurrent novae & -0.25 & -0.9 & 480 \\
\hline Nova-like U Geminorum type stars & -0.27 & $(-0 \cdot 17)$ & 2600 \\
\hline
\end{tabular}

